\title{
A "turn-off" supramolecular fluorescence array sensor for heavy metal ion identification
}

Qin Wanga, Kai-Ni Weia , Shu-Zhen Huang ${ }^{\mathrm{a}}$, Qing Tang ${ }^{\mathrm{c}}$, Zhu Tao ${ }^{\mathrm{a}}$,Ying Huanga,b*

${ }^{a}$ Key Laboratory of Macrocyclic and Supramolecular Chemistry of Guizhou Province, Guizhou University, Guiyang 550025, China.

b The Engineering and Research Center for Southwest Bio-Pharmaceutical Resources of National Education Ministry of China, Guizhou University, Guiyang 550025, China.

${ }^{c}$ Department College of Tobacco Science, Guizhou University, Guiyang 550025, China.

(a)

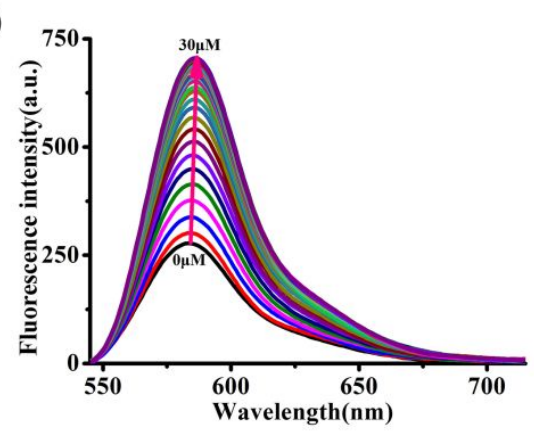

(c)

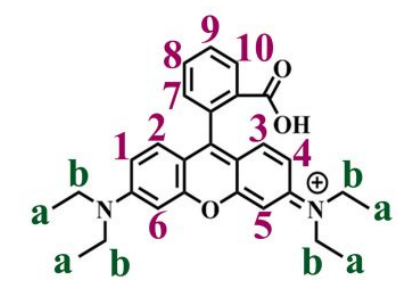

(b)

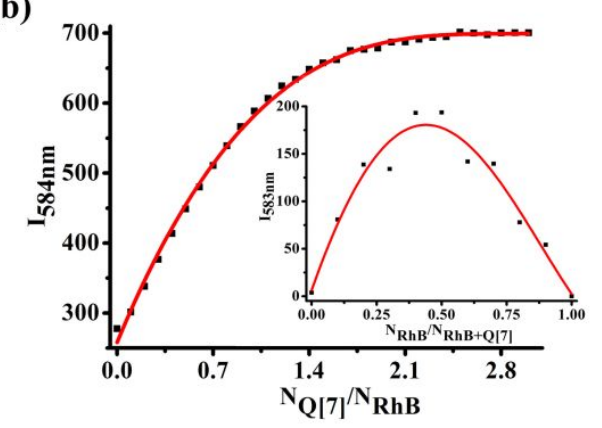

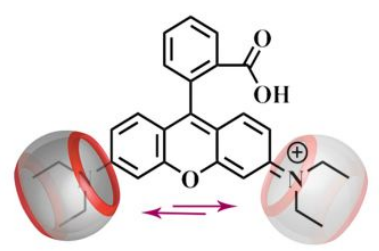

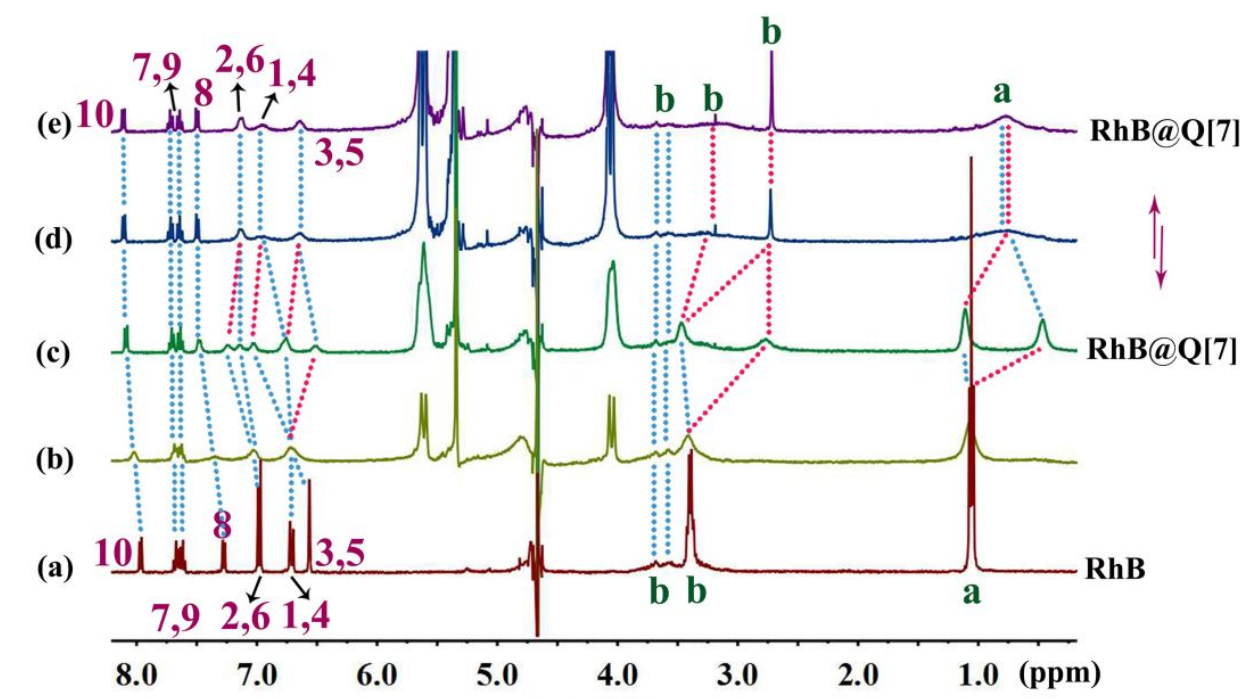


Figure S1 The fluorescence spectra recorded for $\mathrm{RhB}(10 \mu \mathrm{M})$ upon increasing the concentration of Q[7] (a); plots of $\mathrm{N}_{\mathrm{Q}[7]} / \mathrm{N}_{\mathrm{RhB}}$ vs. fluorescence intensity of RhB (b), inserts: Job's plot obtained by continuous variation of the mole fraction of $\mathrm{RhB}$ and $\mathrm{Q}[7]$ (b); the possible interaction modes (top of c) and ${ }^{1} \mathrm{H}$ NMR titration spectra of RhB recorded upon the addition of Q[7](c), respectively.

(a)

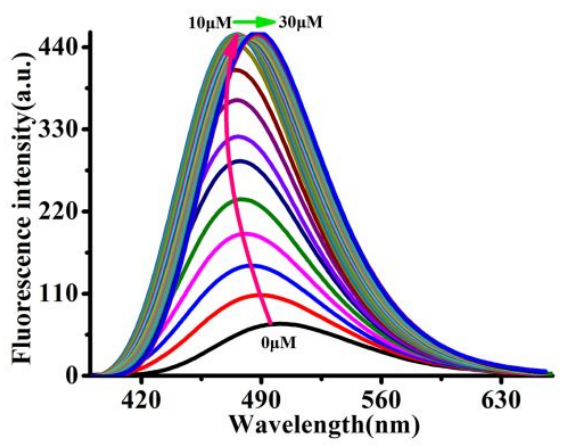

(b)

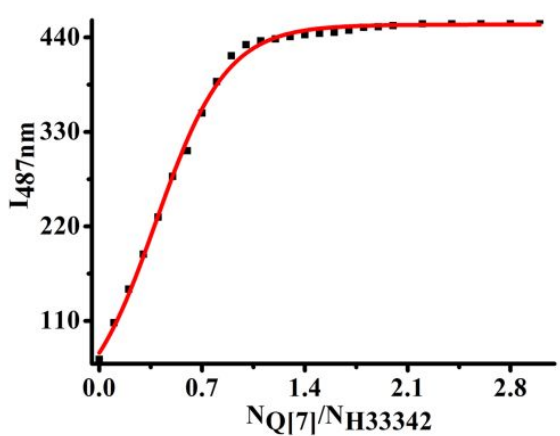

(c)

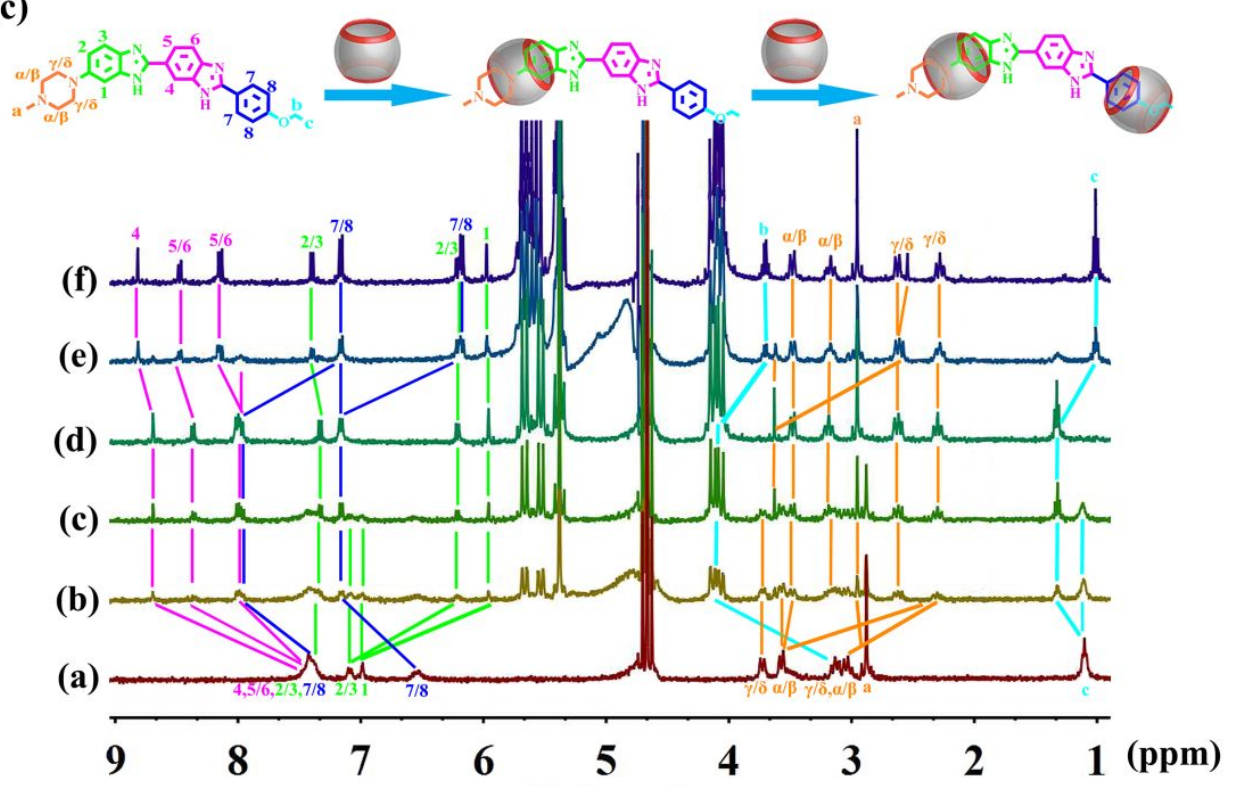

Figure S2 The fluorescence spectra recorded for H33342 (10 $\mu \mathrm{M})$ upon increasing the concentration of Q[7] (a); plots of $\mathrm{N}_{\mathrm{Q}[7]} / \mathrm{N}_{\mathrm{H} 33342}$ vs. fluorescence intensity of $\mathrm{H} 33342$ (b); the possible interaction modes (top of c) and ${ }^{1} \mathrm{H}$ NMR titration spectra of $\mathrm{H} 33342$ recorded upon the addition of $\mathrm{Q}[7](\mathrm{c})$, respectively. 
(a)

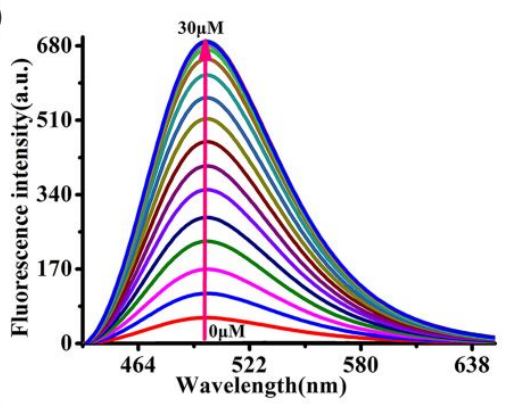

(c)

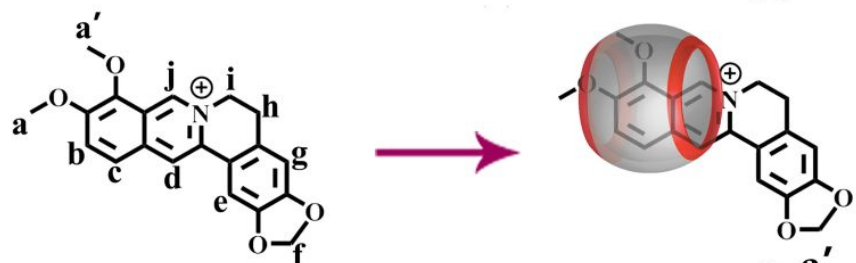

(d)

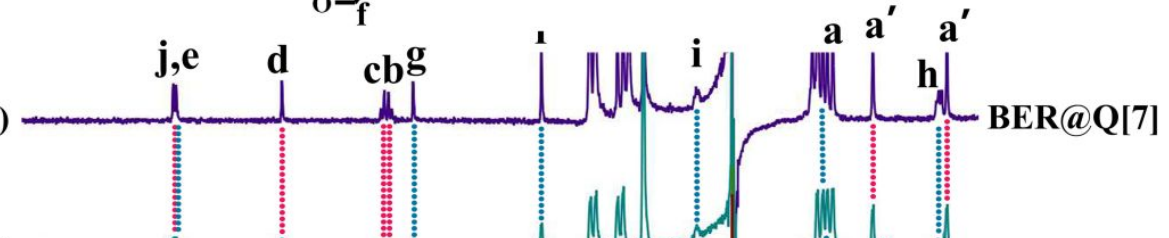

(c)

(b)

(a)

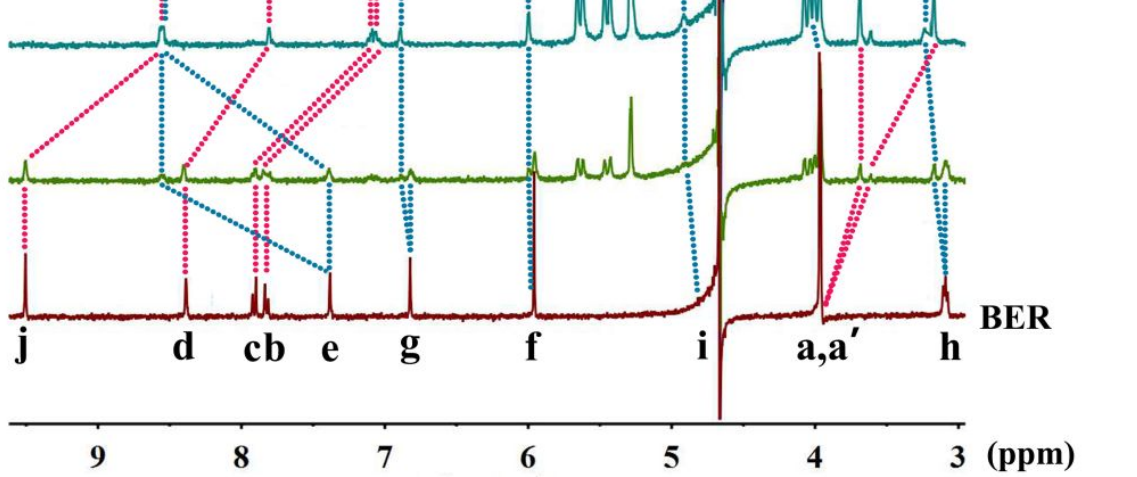

(b)

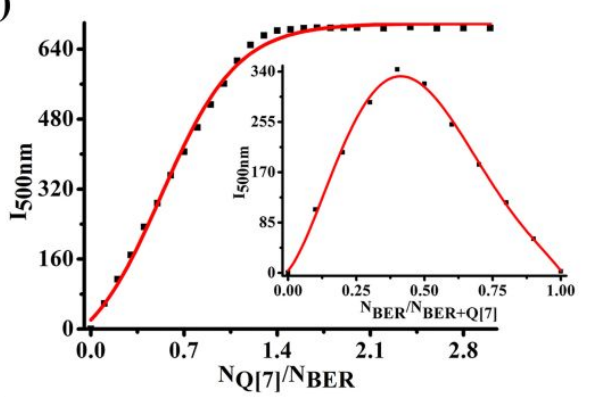

Figure S3 The fluorescence spectra recorded for BER $(10 \mu \mathrm{M})$ upon increasing the concentration of Q[7] (a); plots of $\mathrm{N}_{\mathrm{Q}[7]} \mathrm{N}_{\mathrm{BER}}$ vs. fluorescence intensity of BER (b), inserts: Job's plot obtained by continuous variation of the mole fraction of BER and Q[7] (b); the possible interaction modes (top of c) and ${ }^{1} \mathrm{H}$ NMR titration spectra of BER recorded upon the addition of Q[7](c), respectively. 

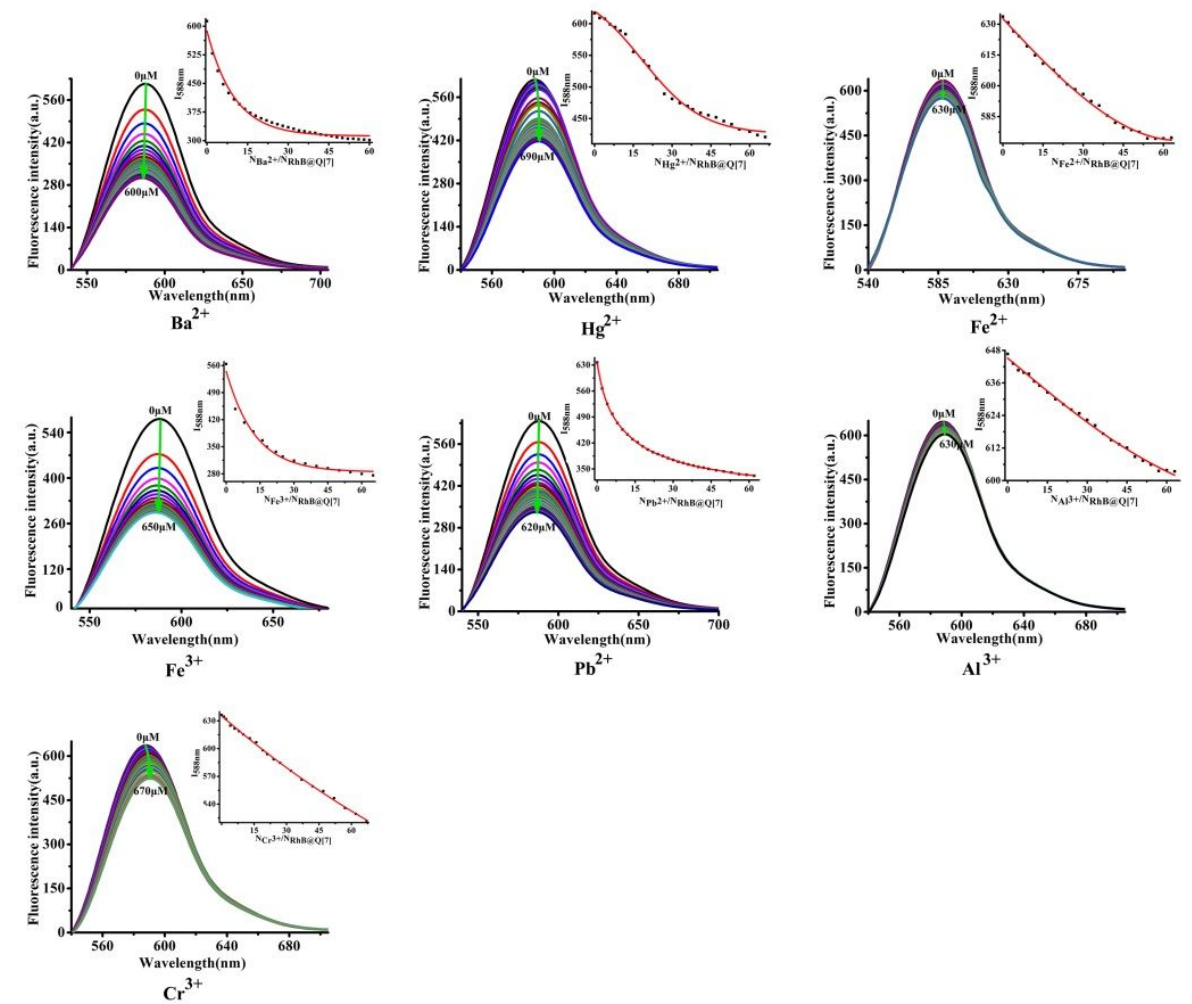

Figure S4 The fluorescence spectra recorded for RhB@Q[7] $(10 \mu \mathrm{M})$ upon increasing the concentration of different metal ions $\left(\mathrm{Ba}^{2+}, \mathrm{Hg}^{2+}, \mathrm{Fe}^{2+}, \mathrm{Fe}^{3+}, \mathrm{Pb}^{2+}, \mathrm{Al}^{3+}, \mathrm{Cr}^{3+}\right)$, inset: plots of $\mathrm{N}_{\mathrm{M}}{ }^{\mathrm{n}} / \mathrm{N}_{\mathrm{RhB} @ \mathrm{Q}[7]}$ vs. fluorescence intensity of RhB@Q[7]. 

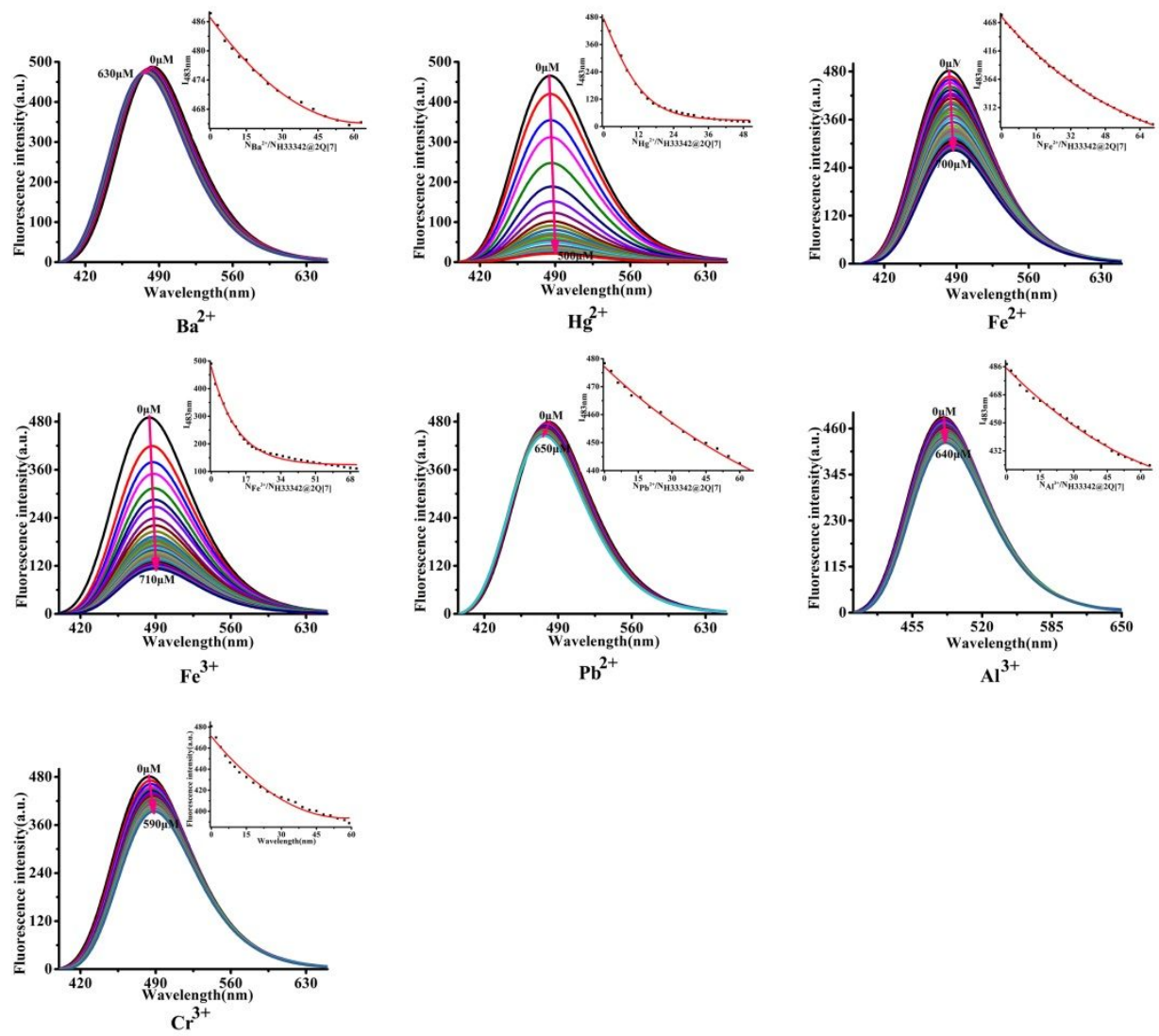

Figure S5 The fluorescence spectra recorded for H33342@2Q[7] $(10 \mu \mathrm{M})$ upon increasing the concentration of different metal ions $\left(\mathrm{Ba}^{2+}, \mathrm{Hg}^{2+}, \mathrm{Fe}^{2+}, \mathrm{Fe}^{3+}, \mathrm{Pb}^{2+}, \mathrm{Al}^{3+}, \mathrm{Cr}^{3+}\right)$, inset: plots of $\mathrm{N}_{\mathrm{M}}{ }^{\mathrm{n}} / \mathrm{N}_{\mathrm{H} 33342 @ 2 \mathrm{Q}[7]}$ vs. fluorescence intensity of H33342@2Q[7]. 

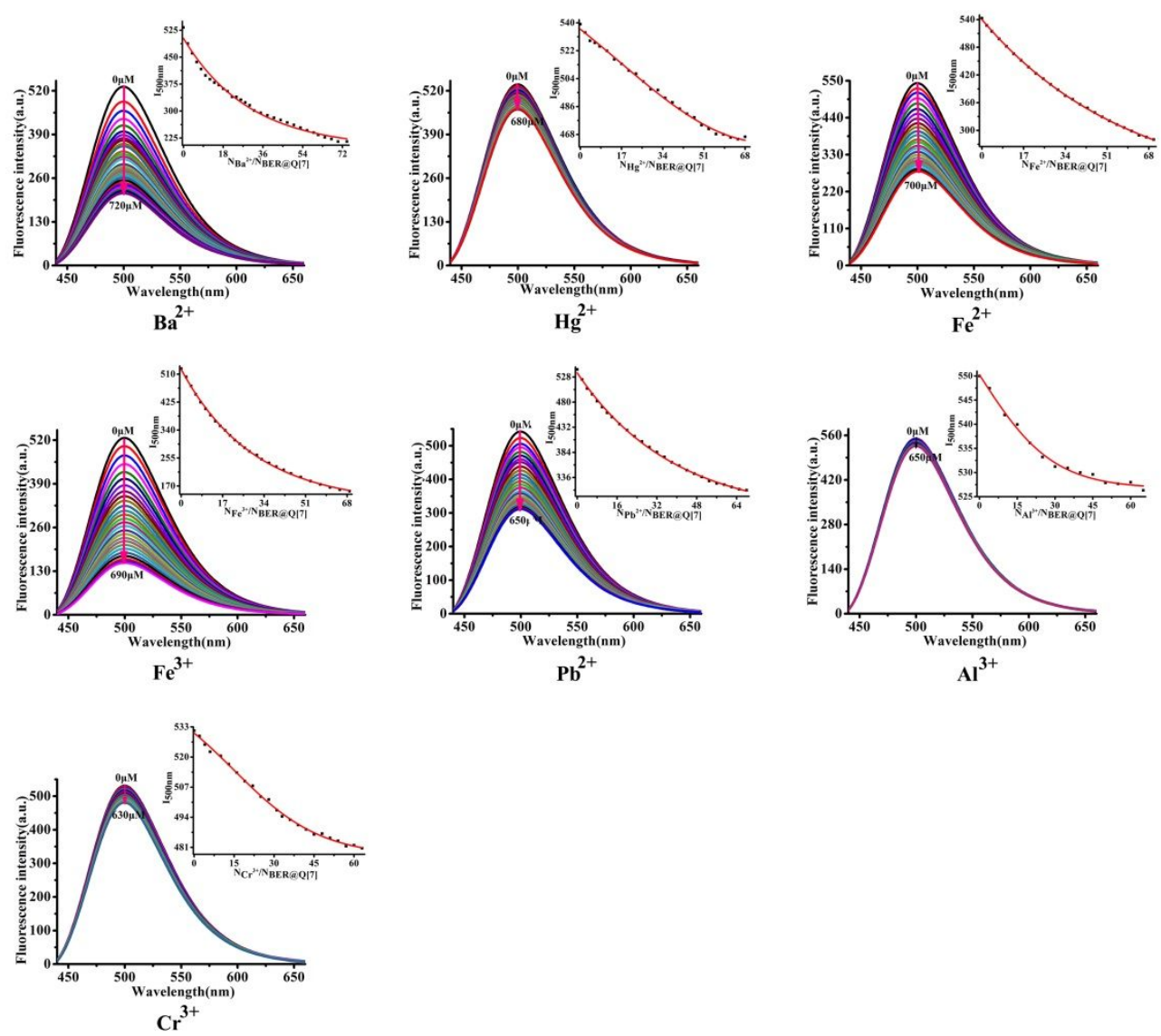

Figure S6 The fluorescence spectra recorded for BER@Q[7] $(10 \mu \mathrm{M})$ upon increasing the concentration of different metal ions $\left(\mathrm{Ba}^{2+}, \mathrm{Hg}^{2+}, \mathrm{Fe}^{2+}, \mathrm{Fe}^{3+}, \mathrm{Pb}^{2+}, \mathrm{Al}^{3+}, \mathrm{Cr}^{3+}\right)$, inset: plots of $\mathrm{N}_{\mathrm{M}}{ }^{\mathrm{n}+} / \mathrm{N}_{\mathrm{BER} @ \mathrm{Q}[7]}$ Vs. fluorescence intensity of BER@Q[7].

Table S1 nonlinear fitting curve for changes in the fluorescence intensity of the inclusion complex in the presence of different metal ions $\left(\mathrm{Ba}^{2+}, \mathrm{Hg}^{2+}, \mathrm{Fe}^{2+}, \mathrm{Fe}^{3+}, \mathrm{Pb}^{2+}, \mathrm{Al}^{3+}, \mathrm{Cr}^{3+}\right)$ of $\mathrm{RhB} @ \mathrm{Q}[7](10 \mu \mathrm{M})$

\begin{tabular}{cccc}
\hline $\mathrm{RhB} @ \mathrm{Q}[7]$ & Correction curve equation & $\mathrm{R}^{2}$ & Limit of detection $(\mathrm{mol} / \mathrm{L})$ \\
\hline $\mathrm{Ba}^{2+}$ & $\Delta \mathrm{I}=22.024 \mathrm{C}+13.956$ & 0.993 & $6.81 \times 10^{-8}$ \\
$\mathrm{Hg}^{2+}$ & $\Delta \mathrm{I}=2.726 \mathrm{C}+0.340$ & 0.995 & $5.50 \times 10^{-7}$ \\
$\mathrm{Fe}^{2+}$ & $\Delta \mathrm{I}=1.417 \mathrm{C}+0.852$ & 0.994 & $1.06 \times 10^{-6}$ \\
$\mathrm{Fe}^{3+}$ & $\Delta \mathrm{I}=1.957 \mathrm{C}+0.273$ & 0.995 & $7.66 \times 10^{-7}$ \\
$\mathrm{~Pb}^{2+}$ & $\Delta \mathrm{I}=17.389 \mathrm{C}+13.973$ & 0.991 & $8.63 \times 10^{-8}$ \\
$\mathrm{Al}^{3+}$ & $\Delta \mathrm{I}=0.860 \mathrm{C}+1.091$ & 0.995 & $1.74 \times 10^{-6}$ \\
$\mathrm{Cr}^{3+}$ & $\Delta \mathrm{I}=0.860 \mathrm{C}+1.091$ & 0.992 & $7.61 \times 10^{-7}$ \\
\hline
\end{tabular}


Table S2 nonlinear fitting curve for changes in the fluorescence intensity of the inclusion complex in the presence of different metal ions $\left(\mathrm{Ba}^{2+}, \mathrm{Hg}^{2+}, \mathrm{Fe}^{2+}, \mathrm{Fe}^{3+}, \mathrm{Pb}^{2+}, \mathrm{Al}^{3+}, \mathrm{Cr}^{3+}\right)$ of H33342@2Q[7] (10 $\left.\mu \mathrm{M}\right)$

\begin{tabular}{llll}
\hline $\mathrm{H} 33342 @ 2 \mathrm{Q}[7]$ & Correction curve equation & $\mathrm{R}^{2}$ & Limit of detection $(\mathrm{mol} / \mathrm{L})$ \\
\hline $\mathrm{Ba}^{2+}$ & $\Delta \mathrm{I}=0.638 \mathrm{C}+0.634$ & 0.990 & $2.35 \times 10^{-6}$ \\
$\mathrm{Hg}^{2+}$ & $\Delta \mathrm{I}=23.427 \mathrm{C}+9.730$ & 0.995 & $6.40 \times 10^{-8}$ \\
$\mathrm{Fe}^{2+}$ & $\Delta \mathrm{I}=4.866 \mathrm{C}+1.982$ & 0.995 & $3.08 \times 10^{-7}$ \\
$\mathrm{Fe}^{3+}$ & $\Delta \mathrm{I}=20.831 \mathrm{C}+16.264$ & 0.991 & $7.20 \times 10^{-8}$ \\
$\mathrm{~Pb}^{2+}$ & $\Delta \mathrm{I}=0.760 \mathrm{C}+0.763$ & 0.992 & $1.97 \times 10^{-6}$ \\
$\mathrm{Al}^{3+}$ & $\Delta \mathrm{I}=1.847 \mathrm{C}+0.677$ & 0.993 & $8.12 \times 10^{-7}$ \\
$\mathrm{Cr}^{3+}$ & $\Delta \mathrm{I}=3.952 \mathrm{C}+2.255$ & 0.992 & $3.80 \times 10^{-7}$ \\
\hline
\end{tabular}

Table S3 nonlinear fitting curve for changes in the fluorescence intensity of the inclusion complex in the presence of different metal ions $\left(\mathrm{Ba}^{2+}, \mathrm{Hg}^{2+}, \mathrm{Fe}^{2+}, \mathrm{Fe}^{3+}, \mathrm{Pb}^{2+}, \mathrm{Al}^{3+}, \mathrm{Cr}^{3+}\right)$ of BER@Q[7] (10 $\left.\mu \mathrm{M}\right)$

\begin{tabular}{cccc}
\hline BER@Q[7] & Correction curve equation & $\mathrm{R}^{2}$ & Limit of detection $(\mathrm{mol} / \mathrm{L})$ \\
\hline $\mathrm{Ba}^{2+}$ & $\Delta \mathrm{I}=13.481 \mathrm{C}+9.453$ & 0.991 & $2.23 \times 10^{-7}$ \\
$\mathrm{Hg}^{2+}$ & $\Delta \mathrm{I}=1.456 \mathrm{C}+1.861$ & 0.991 & $2.06 \times 10^{-6}$ \\
$\mathrm{Fe}^{2+}$ & $\Delta \mathrm{I}=5.471 \mathrm{C}+4.403$ & 0.997 & $5.48 \times 10^{-7}$ \\
$\mathrm{Fe}^{3+}$ & $\Delta \mathrm{I}=11.506 \mathrm{C}+4.449$ & 0.997 & $2.61 \times 10^{-7}$ \\
$\mathrm{~Pb}^{2+}$ & $\Delta \mathrm{I}=6.380 \mathrm{C}+6.432$ & 0.992 & $4.70 \times 10^{-7}$ \\
$\mathrm{Al}^{3+}$ & $\Delta \mathrm{I}=0.644 \mathrm{C}+0.374$ & 0.995 & $4.66 \times 10^{-6}$ \\
$\mathrm{Cr}^{3+}$ & $\Delta \mathrm{I}=1.093 \mathrm{C}+0.780$ & 0.992 & $2.75 \times 10^{-6}$ \\
\hline
\end{tabular}



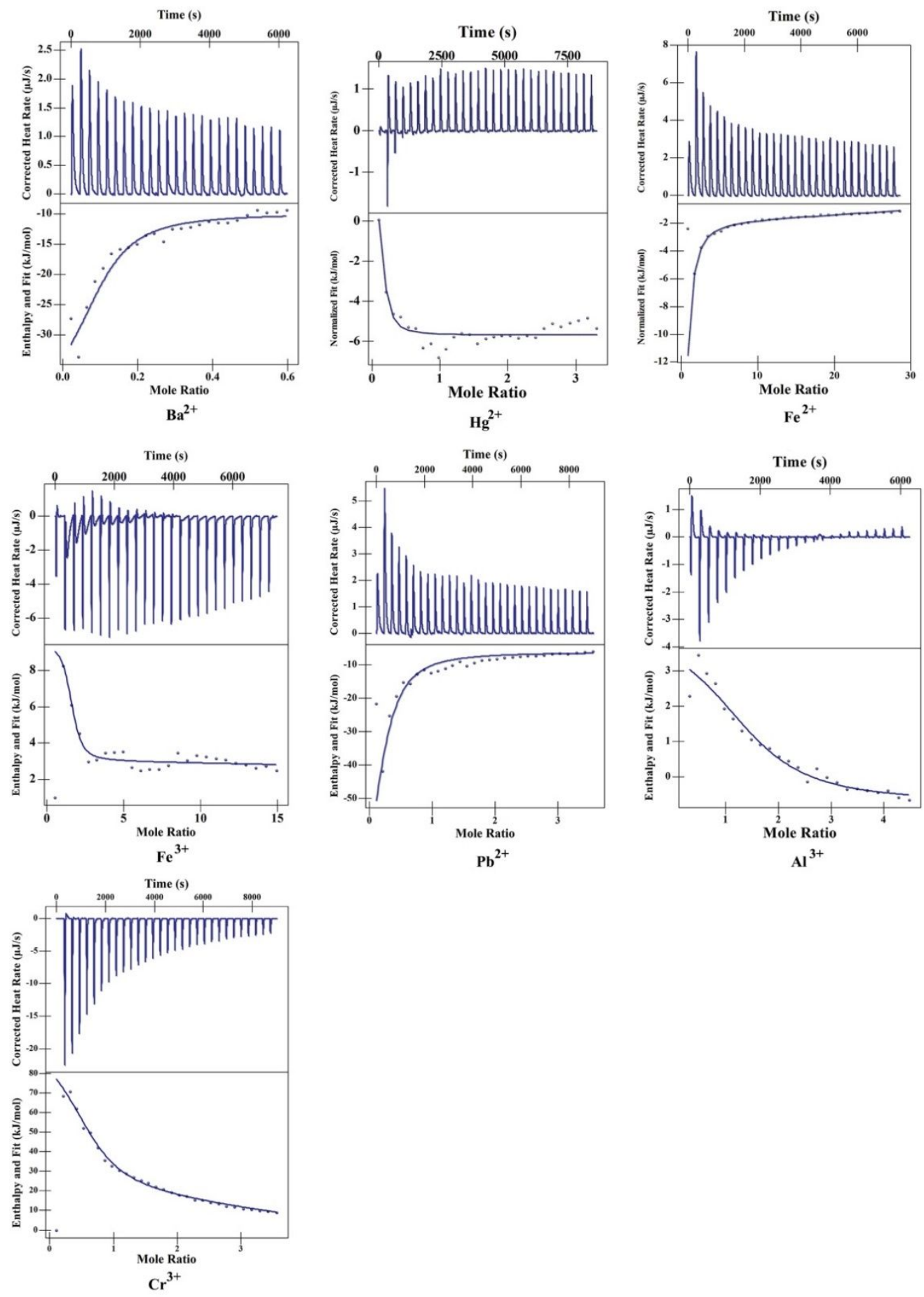

Figure S7 ITC curves $(298 \mathrm{~K})$ of $\mathrm{RhB} @ \mathrm{Q}[7]\left(1 \times 10^{-4} \mathrm{M}\right)$ into different metal ions $\left(\mathrm{Ba}^{2+}, \mathrm{Hg}^{2+}, \mathrm{Fe}^{2+}, \mathrm{Fe}^{3+}\right.$, $\left.\mathrm{Pb}^{2+}, \mathrm{Al}^{3+}, \mathrm{Cr}^{3+}, 1.5 \times 10^{-3} \mathrm{M}\right)$ 

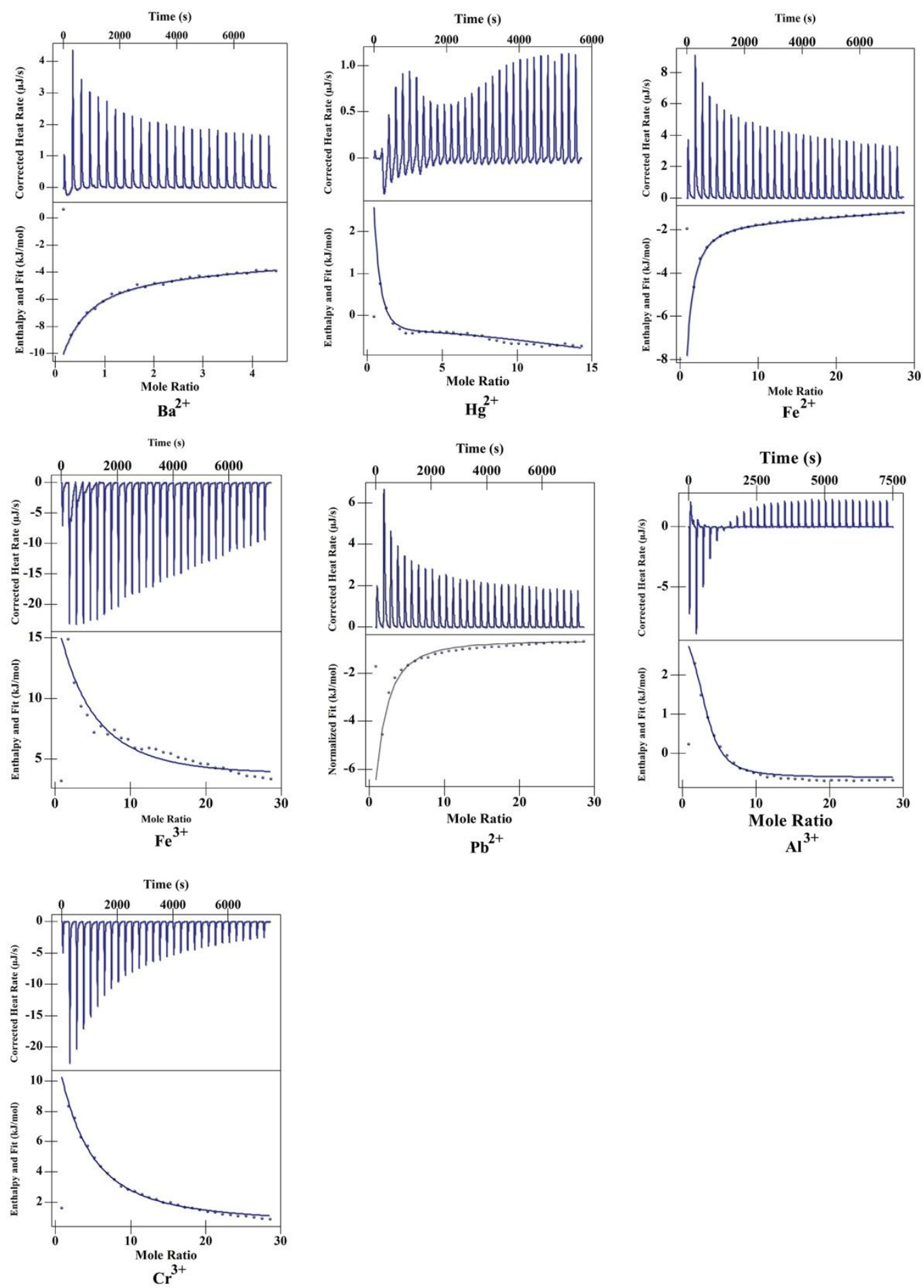

Figure S8 ITC curves $(298 \mathrm{~K})$ of H33342@2Q[7] $\left(1 \times 10^{-4} \mathrm{M}\right)$ into different metal ions $\left(\mathrm{Ba}^{2+}, \mathrm{Hg}^{2+}, \mathrm{Fe}^{2+}, \mathrm{Fe}^{3+}\right.$, $\left.\mathrm{Pb}^{2+}, \mathrm{Al}^{3+}, \mathrm{Cr}^{3+}, 1.5 \times 10^{-3} \mathrm{M}\right)$. 

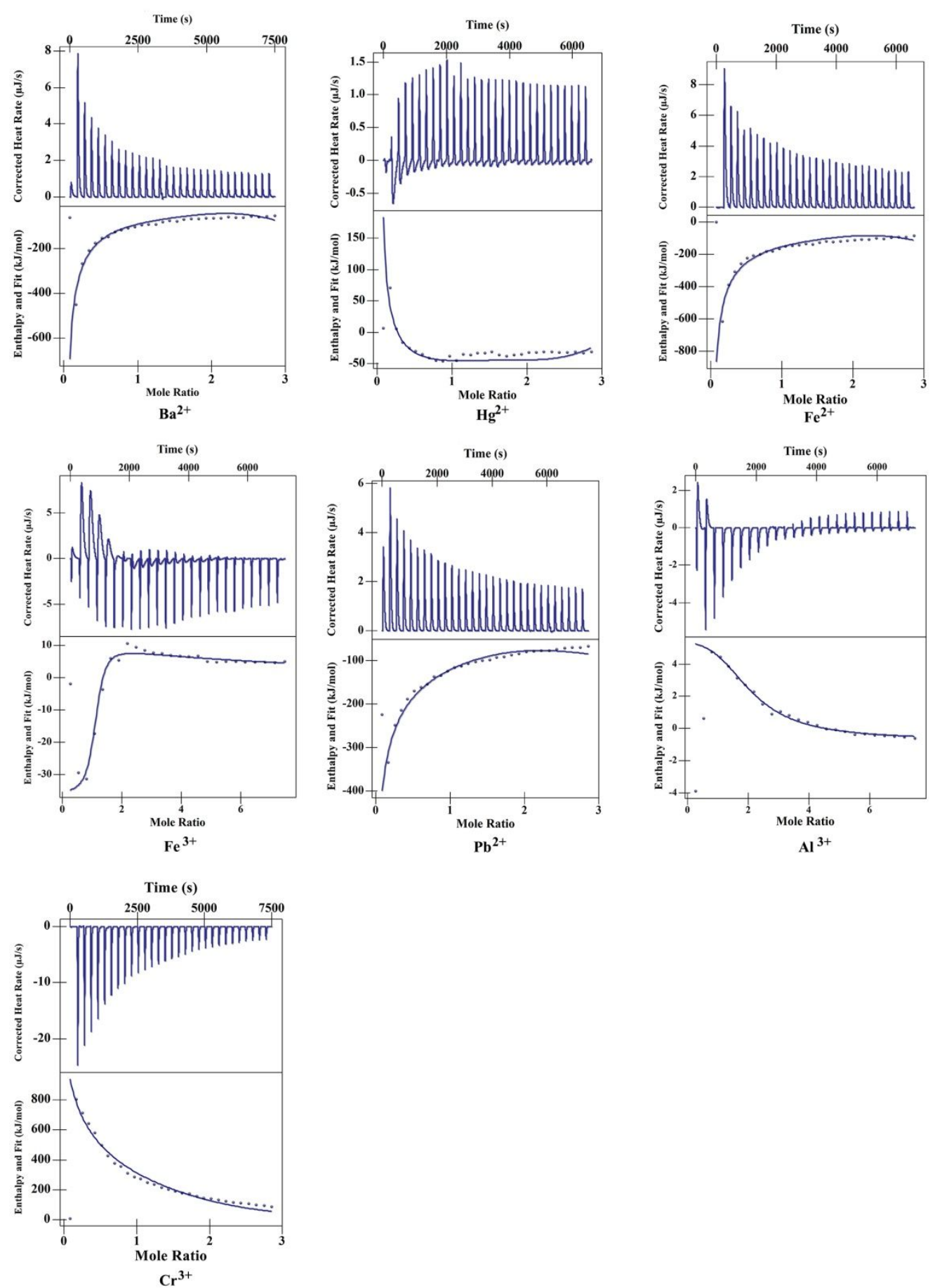

Figure S9 ITC curves $(298 \mathrm{~K})$ of BER@Q[7] $\left(1 \times 10^{-4} \mathrm{M}\right)$ into different metal ions $\left(\mathrm{Ba}^{2+}, \mathrm{Hg}^{2+}, \mathrm{Fe}^{2+}, \mathrm{Fe}^{3+}\right.$, $\left.\mathrm{Pb}^{2+}, \mathrm{Al}^{3+}, \mathrm{Cr}^{3+}, 1.5 \times 10^{-3} \mathrm{M}\right)$

Table S4 The binding constant and thermodynamic parameter (298 K)for RhB@Q[7] with different metal ions $\left(\mathrm{Ba}^{2+}, \mathrm{Hg}^{2+}, \mathrm{Fe}^{2+}, \mathrm{Fe}^{3+}, \mathrm{Pb}^{2+}, \mathrm{Al}^{3+}\right)$ 


\begin{tabular}{cccc}
\hline $\mathrm{RhB} @ \mathrm{Q}[7]$ & $\mathrm{K}\left(\mathrm{M}^{-1}\right)$ & $\Delta \mathrm{H}(\mathrm{KJ} / \mathrm{mol})$ & $\Delta \mathrm{S}(\mathrm{J} / \mathrm{mol} . \mathrm{K})$ \\
\hline $\mathrm{Ba}^{2+}$ & $1.29 \times 10^{5}$ & $-0.32 \times 10^{2}$ & $-0.082 \times 10^{2}$ \\
$\mathrm{Hg}^{2+}$ & $3.02 \times 10^{5}$ & $0.11 \times 10^{2}$ & $1.42 \times 10^{2}$ \\
$\mathrm{Fe}^{2+}$ & $1.14 \times 10^{4}$ & $-0.10 \times 10^{2}$ & $-2.58 \times 10^{2}$ \\
$\mathrm{Fe}^{3+}$ & $1.33 \times 10^{5}$ & $0.064 \times 10^{2}$ & $1.20 \times 10^{2}$ \\
$\mathrm{~Pb}^{2+}$ & $6.44 \times 10^{4}$ & $-1.00 \times 10^{2}$ & $-2.43 \times 10^{2}$ \\
$\mathrm{Al}^{3+}$ & $2.84 \times 10^{4}$ & $0.050 \times 10^{2}$ & $1.02 \times 10^{2}$ \\
$\mathrm{Cr}^{3}$ & $6.93 \times 10^{4}$ & $0.070 \times 10^{2}$ & $3.26 \times 10^{2}$ \\
\hline
\end{tabular}

Table S5 The binding constant and thermodynamic parameter (298 K)for H33342@2Q[7] with different metal ions $\left(\mathrm{Ba}^{2+}, \mathrm{Hg}^{2+}, \mathrm{Fe}^{2+}, \mathrm{Fe}^{3+}, \mathrm{Pb}^{2+}, \mathrm{Al}^{3+}\right)$

\begin{tabular}{cccc}
\hline $\mathrm{H} 33342 @ 2 \mathrm{Q}[7]$ & $\mathrm{K}\left(\mathrm{M}^{-1}\right)$ & $\Delta \mathrm{H}(\mathrm{KJ} / \mathrm{mol})$ & $\Delta \mathrm{S}(\mathrm{J} / \mathrm{mol} . \mathrm{K})$ \\
\hline $\mathrm{Ba}^{2+}$ & $1.11 \times 10^{4}$ & $-0.61 \times 10^{2}$ & $-1.27 \times 10^{2}$ \\
$\mathrm{Hg}^{2+}$ & $\mathrm{K}_{1}=2.82 \times 10^{4}$ & $\Delta \mathrm{H}_{1}=0.090 \times 10^{2}$ & $\Delta \mathrm{S}_{1}=1.17 \times 10^{2}$ \\
& $\mathrm{~K}_{2}=1.00 \times 10^{3}$ & $\Delta \mathrm{H}_{2}=-0.91 \times 10^{2}$ & $\Delta \mathrm{S}_{2}=-2.49 \times 10^{2}$ \\
$\mathrm{Fe}^{2+}$ & $7.23 \times 10^{3}$ & $-1.00 \times 10^{2}$ & $-2.61 \times 10^{2}$ \\
$\mathrm{Fe}^{3+}$ & $1.21 \times 10^{3}$ & $1.00 \times 10^{2}$ & $3.94 \times 10^{2}$ \\
$\mathrm{~Pb}^{2+}$ & $3.75 \times 10^{3}$ & $-1.00 \times 10^{2}$ & $-2.67 \times 10^{2}$ \\
$\mathrm{Al}^{3+}$ & $1.55 \times 10^{4}$ & $0.065 \times 10^{2}$ & $1.02 \times 10^{2}$ \\
$\mathrm{Cr}^{3}$ & $1.34 \times 10^{3}$ & $1.00 \times 10^{2}$ & $3.95 \times 10^{2}$ \\
\hline
\end{tabular}

Table S6 The binding constant and thermodynamic parameter (298 K)for BER@Q[7] with different metal ions $\left(\mathrm{Ba}^{2+}, \mathrm{Hg}^{2+}, \mathrm{Fe}^{2+}, \mathrm{Fe}^{3+}, \mathrm{Pb}^{2+}, \mathrm{Al}^{3+}\right)$

\begin{tabular}{|c|c|c|c|}
\hline BER@Q[7] & $\mathrm{K}\left(\mathrm{M}^{-1}\right)$ & $\Delta \mathrm{H}(\mathrm{KJ} / \mathrm{mol})$ & $\Delta \mathrm{S}(\mathrm{J} / \mathrm{mol} . \mathrm{K})$ \\
\hline \multirow[t]{2}{*}{$\mathrm{Ba}^{2+}$} & $\mathrm{K}_{1}=3.67 \times 10^{4}$ & $\Delta \mathrm{H}_{1}=-5.39 \times 10^{3}$ & $\Delta \mathrm{S}_{1}=-1.80 \times 10^{4}$ \\
\hline & $\mathrm{K}_{2}=3.21 \times 10^{6}$ & $\Delta \mathrm{H}_{2}=5.00 \times 10^{3}$ & $\Delta S_{2}=1.69 \times 10^{4}$ \\
\hline
\end{tabular}




\begin{tabular}{|c|c|c|c|}
\hline \multirow[t]{2}{*}{$\mathrm{Hg}^{2+}$} & $\mathrm{K}_{1}=1.66 \times 10^{4}$ & $\Delta \mathrm{H}_{1}=5.25 \times 10^{3}$ & $\Delta \mathrm{S}_{1}=1.76 \times 10^{4}$ \\
\hline & $\mathrm{K}_{2}=9.43 \times 10^{6}$ & $\Delta \mathrm{H}_{2}=-5.00 \times 10^{3}$ & $\Delta \mathrm{S}_{2}=-1.66 \times 10^{4}$ \\
\hline \multirow[t]{2}{*}{$\mathrm{Fe}^{2+}$} & $\mathrm{K}_{1}=4.07 \times 10^{4}$ & $\Delta \mathrm{H}_{1}=-5.41 \times 10^{3}$ & $\Delta \mathrm{S}_{1}=-1.81 \times 10^{4}$ \\
\hline & $\mathrm{K}_{2}=2.33 \times 10^{6}$ & $\Delta \mathrm{H}_{2}=5.00 \times 10^{3}$ & $\Delta S_{2}=1.69 \times 10^{4}$ \\
\hline \multirow[t]{2}{*}{$\mathrm{Fe}^{3+}$} & $\mathrm{K}_{1}=4.17 \times 10^{5}$ & $\Delta \mathrm{H}_{1}=-0.36 \times 10^{2}$ & $\Delta \mathrm{S}_{1}=-0.014 \times 10^{2}$ \\
\hline & $\mathrm{K} 2=5.64 \times 10^{2}$ & $\Delta \mathrm{H}_{2}=2.51 \times 10^{2}$ & $\Delta \mathrm{S}_{2}=8.92 \times 10^{2}$ \\
\hline \multirow[t]{2}{*}{$\mathrm{Pb}^{2+}$} & $\mathrm{K}_{1}=7.76 \times 10^{4}$ & $\Delta \mathrm{H}_{1}=-7.73 \times 10^{2}$ & $\Delta \mathrm{S}_{1}=-2.50 \times 10^{3}$ \\
\hline & $\mathrm{K}_{2}=5.81 \times 10^{5}$ & $\Delta \mathrm{H}_{2}=9.50 \times 10^{2}$ & $\Delta \mathrm{S}_{2}=3.30 \times 10^{3}$ \\
\hline \multirow[t]{2}{*}{$\mathrm{Al}^{3+}$} & $\mathrm{K}_{1}=8.49 \times 10^{4}$ & $\Delta \mathrm{H}_{1}=0.067 \times 10^{2}$ & $\Delta \mathrm{S}_{1}=1.17 \times 10^{2}$ \\
\hline & $\mathrm{K} 2=9.82 \times 10^{3}$ & $\Delta \mathrm{H}_{2}=0.096 \times 10^{2}$ & $\Delta \mathrm{S}_{2}=1.08 \times 10^{2}$ \\
\hline \multirow[t]{2}{*}{$\mathrm{Cr}^{3}$} & $\mathrm{~K}_{1}=2.31 \times 10^{4}$ & $\Delta \mathrm{H}_{1}=5.23 \times 10^{3}$ & $\Delta \mathrm{S}_{1}=1.76 \times 10^{4}$ \\
\hline & $\mathrm{K}_{2}=4.95 \times 10^{5}$ & $\Delta \mathrm{H}_{2}=-5.00 \times 10^{3}$ & $\Delta S_{2}=-1.67 \times 10^{4}$ \\
\hline
\end{tabular}

Table S7 Fluorescence response patterns generated from three sensor arrays against seven different metal ions (500 $\mu \mathrm{M}$ metal, $10 \mu \mathrm{M}$ sensor in ultra-pure water).

Fold-change in fluorescence $(F)$

\begin{tabular}{cccc}
\hline & BER@Q[7] & H33342@2Q[7] & RHB@Q[7] \\
$\mathrm{Ba}^{2+}$ & 0.0358 & 0.9060 & 0.3580 \\
\cline { 2 - 3 } $\mathrm{Ba}^{2+}$ & 0.0222 & 0.9306 & 0.3412 \\
$\mathrm{Ba}^{2+}$ & 0.0401 & 0.9178 & 0.2780 \\
$\mathrm{Ba}^{2+}$ & 0.0473 & 0.8752 & 0.3624 \\
$\mathrm{Ba}^{2+}$ & 0.0315 & 0.8937 & 0.3974 \\
$\mathrm{Hg}^{2+}$ & 0.8133 & -0.1752 & 0.1721 \\
$\mathrm{Hg}^{2+}$ & 0.8376 & -0.1701 & 0.2519 \\
$\mathrm{Hg}^{2+}$ & 0.8047 & -0.1583 & 0.2280 \\
$\mathrm{Hg}^{2+}$ & 0.7595 & -0.1655 & 0.2269 \\
$\mathrm{Hg}^{2+}$ & 0.7588 & -0.1709 & 0.3138
\end{tabular}




\begin{tabular}{|c|c|c|c|}
\hline $\mathrm{Fe}^{2+}$ & 0.6341 & 0.2650 & 0.5896 \\
\hline $\mathrm{Fe}^{2+}$ & 0.6083 & 0.2158 & 0.5795 \\
\hline $\mathrm{Fe}^{2+}$ & 0.6535 & 0.1532 & 0.5752 \\
\hline $\mathrm{Fe}^{2+}$ & 0.6506 & 0.2296 & 0.5600 \\
\hline $\mathrm{Fe}^{2+}$ & 0.6320 & 0.2296 & 0.5752 \\
\hline $\mathrm{Fe}^{3+}$ & 0.2443 & -0.0023 & 0.3184 \\
\hline $\mathrm{Fe}^{3+}$ & 0.2931 & -0.0190 & 0.3105 \\
\hline $\mathrm{Fe}^{3+}$ & 0.2458 & 0.0226 & 0.3331 \\
\hline $\mathrm{fe}^{3+}$ & 0.2916 & -0.0334 & 0.3371 \\
\hline $\mathrm{fe}^{3+}$ & 0.2680 & 0.0659 & 0.3700 \\
\hline $\mathrm{Pb}^{2+}$ & 0.0423 & 0.8726 & 0.4023 \\
\hline $\mathrm{Pb}^{2+}$ & 0.0459 & 0.8780 & 0.3151 \\
\hline $\mathrm{Pb}^{2+}$ & 0.0287 & 0.8547 & 0.3890 \\
\hline $\mathrm{Pb}^{2+}$ & 0.0523 & 0.8837 & 0.3635 \\
\hline $\mathrm{Pb}^{2+}$ & 0.0487 & 0.8660 & 0.3122 \\
\hline $\mathrm{Al}^{3+}$ & 0.9551 & 0.6522 & 0.9680 \\
\hline $\mathrm{Al}^{3+}$ & 0.9129 & 0.6712 & 0.9161 \\
\hline $\mathrm{Al}^{3+}$ & 0.9372 & 0.6715 & 0.9259 \\
\hline $\mathrm{Al}^{3+}$ & 0.9093 & 0.6692 & 1.0052 \\
\hline $\mathrm{Al}^{3+}$ & 0.9265 & 0.5175 & 0.9498 \\
\hline $\mathrm{Cr}^{3+}$ & 0.8656 & 0.3602 & 0.6561 \\
\hline $\mathrm{Cr}^{3+}$ & 0.8763 & 0.4177 & 0.6990 \\
\hline $\mathrm{Cr}^{3+}$ & 0.8512 & 0.4057 & 0.6094 \\
\hline $\mathrm{Cr}^{3+}$ & 0.8706 & 0.3856 & 0.6355 \\
\hline $\mathrm{Cr}^{3+}$ & 0.7868 & 0.3980 & 0.6498 \\
\hline
\end{tabular}

Table S8 LDA classification characteristic values of three sensor array against seven different metal ions $(500 \mu \mathrm{M}$ metal, $10 \mu \mathrm{M}$ sensor in ultra-pure water $)$ 


\begin{tabular}{ccccc}
\hline function & eigenvalues & Variation(\%) & Cumulative(\%) & Canonical correlation \\
\hline 1 & 478.464 & 68.6 & 68.6 & .999 \\
2 & 210.057 & 30.1 & 98.7 & .998 \\
3 & 8.755 & 1.3 & 100.0 & .947 \\
\hline
\end{tabular}

Table S9 Training matrix of fluorescence response patterns generated from three sensor arrays against seven different metal ions (500 $\mu \mathrm{M}$ metal, $10 \mu \mathrm{M}$ sensor in ultra-pure water, red cells indicate false classifications)

\begin{tabular}{|c|c|c|c|c|c|c|c|c|c|c|}
\hline \multirow[t]{2}{*}{ Metal } & \multicolumn{10}{|c|}{ Predictive classification } \\
\hline & & & $\mathrm{Ba}^{2+}$ & $\mathrm{Hg}^{2+}$ & $\mathrm{Fe}^{2+}$ & $\mathrm{Fe}^{3+}$ & $\mathrm{Pb}^{2+}$ & $\mathrm{Al}^{3+}$ & $\mathrm{Cr}^{3+}$ & total \\
\hline \multirow[t]{7}{*}{ Original } & count & $\mathrm{Ba}^{2+}$ & 3 & 0 & 0 & 0 & 2 & 0 & 0 & 5 \\
\hline & & $\mathrm{Hg}^{2+}$ & 0 & 5 & 0 & 0 & 0 & 0 & 0 & 5 \\
\hline & & $\mathrm{Fe}^{2+}$ & 0 & 0 & 5 & 0 & 0 & 0 & 0 & 5 \\
\hline & & $\mathrm{Fe}^{3+}$ & 0 & 0 & 0 & 5 & 0 & 0 & 0 & 5 \\
\hline & & $\mathrm{Pb}^{2+}$ & 1 & 0 & 0 & 0 & 4 & 0 & 0 & 5 \\
\hline & & $\mathrm{Al}^{3+}$ & 0 & 0 & 0 & 0 & 0 & 5 & 0 & 5 \\
\hline & & $\mathrm{Cr}^{3+}$ & 0 & 0 & 0 & 0 & 0 & 0 & 5 & 5 \\
\hline \multirow[t]{7}{*}{ Cross-validated } & count & $\mathrm{Ba}^{2+}$ & 3 & 0 & 0 & 0 & 2 & 0 & 0 & 5 \\
\hline & & $\mathrm{Hg}^{2+}$ & 0 & 5 & 0 & 0 & 0 & 0 & 0 & 5 \\
\hline & & $\mathrm{Fe}^{2+}$ & 0 & 0 & 5 & 0 & 0 & 0 & 0 & 5 \\
\hline & & $\mathrm{Fe}^{3+}$ & 0 & 0 & 0 & 5 & 0 & 0 & 0 & 5 \\
\hline & & $\mathrm{Pb}^{2+}$ & 2 & 0 & 0 & 0 & 3 & 0 & 0 & 5 \\
\hline & & $\mathrm{Al}^{3+}$ & 0 & 0 & 0 & 0 & 0 & 5 & 0 & 5 \\
\hline & & $\mathrm{Cr}^{3+}$ & 0 & 0 & 0 & 0 & 0 & 0 & 5 & 5 \\
\hline
\end{tabular}

Table S10 Fluorescence response patterns generated from three sensor arrays against mixed samples of $\mathrm{Pb}^{2+}$ and $\mathrm{Hg}^{2+}$ with different concentrations $\left(\mathrm{C}_{\mathrm{Pb}^{2+}}{ }^{2+} \mathrm{C}_{\mathrm{Hg}}{ }^{2+}=600 \mu \mathrm{M}, 10 \mu \mathrm{M}\right.$ sensor in ultra-pure water $)$ 


\begin{tabular}{|c|c|c|c|}
\hline \multirow{2}{*}{$\mathrm{C}_{\mathrm{Pb}^{2+} / \mathrm{C}_{\mathrm{Hg}}{ }^{2+}(\mu \mathrm{M})}$} & \multicolumn{3}{|c|}{ Fold-change in fluorescence $(\mathrm{F})$} \\
\hline & BER@Q[7] & H33342@2Q[7] & RhB@Q[7] \\
\hline $0: 600$ & 0.7073 & -0.1395 & 0.1927 \\
\hline $0: 600$ & 0.7036 & -0.1425 & 0.1927 \\
\hline $0: 600$ & 0.6100 & -0.1365 & 0.1173 \\
\hline $0: 600$ & 0.6842 & -0.1423 & 0.1016 \\
\hline $0: 600$ & 0.6568 & -0.1443 & 0.1327 \\
\hline 100:500 & 0.2340 & -0.1420 & -0.0307 \\
\hline $100: 500$ & 0.2689 & -0.1393 & -0.0863 \\
\hline $100: 500$ & 0.1724 & -0.1355 & -0.0808 \\
\hline $100: 500$ & 0.3061 & -0.1363 & -0.0014 \\
\hline $100: 500$ & 0.2385 & -0.1390 & -0.0266 \\
\hline $300: 300$ & 0.0535 & -0.1028 & -0.0788 \\
\hline $300: 300$ & 0.0698 & -0.1186 & -0.0096 \\
\hline $300: 300$ & 0.0795 & -0.1266 & -0.0965 \\
\hline $300: 300$ & 0.0297 & -0.1168 & -0.1092 \\
\hline $300: 300$ & 0.0431 & -0.1156 & -0.0583 \\
\hline 500:100 & 0.0357 & 0.2079 & 0.0733 \\
\hline $500: 100$ & 0.0037 & 0.1955 & -0.1143 \\
\hline 500:100 & 0.0067 & 0.1805 & 0.0539 \\
\hline 500:100 & 0.0045 & 0.0841 & 0.0160 \\
\hline $500: 100$ & 0.0379 & -0.0584 & -0.0102 \\
\hline 600:0 & -0.0022 & 1.0132 & 0.0443 \\
\hline $600: 0$ & 0.0164 & 0.9675 & 0.0464 \\
\hline 600:0 & 0.0156 & 0.9643 & -0.0017 \\
\hline 600:0 & 0.0282 & 1.0022 & 0.0652 \\
\hline $600: 0$ & 0.0371 & 0.9800 & 0.0160 \\
\hline
\end{tabular}


Table S11 LDA classification characteristic values of three sensor array against mixed samples of $\mathrm{Pb}^{2+}$ and $\mathrm{Hg}^{2+}$ with different concentrations $\left(\mathrm{C}_{\mathrm{Pb}}{ }^{2+}+\mathrm{C}_{\mathrm{Hg}}{ }^{2+}=600 \mu \mathrm{M}, 10 \mu \mathrm{M}\right.$ sensor in ultra-pure water $)$

\begin{tabular}{ccccc}
\hline function & eigenvalues & Variation(\%) & Cumulative(\%) & Canonical correlation \\
\hline 1 & 281.414 & 70.0 & 70.0 & .998 \\
2 & 111.458 & 27.7 & 97.7 & .996 \\
3 & 9.074 & 2.3 & 100.0 & .949 \\
\hline
\end{tabular}

Table S12 Training matrix of fluorescence response patterns generated from three sensor arrays against mixed samples of $\mathrm{Pb}^{2+}$ and $\mathrm{Hg}^{2+}$ with different concentrations $\left(\mathrm{C}_{\mathrm{Pb}^{2+}}{ }^{2+} \mathrm{C}_{\mathrm{Hg}}{ }^{2+}=600 \mu \mathrm{M}, 10 \mu \mathrm{M}\right.$ sensor in ultra-pure water, red cells indicate false classifications)

\begin{tabular}{|c|c|c|c|c|c|c|c|c|}
\hline \multirow{2}{*}{$\begin{array}{c}\mathrm{C}_{\mathrm{Pb}}^{2+} / \mathrm{C}_{\mathrm{Hg}}{ }^{2+} \\
(\mu \mathrm{M})\end{array}$} & \multicolumn{8}{|c|}{ Predictive classification } \\
\hline & & & $0: \quad 600$ & $100: 500$ & $300: 300$ & $500: 100$ & $600: 0$ & total \\
\hline \multirow[t]{5}{*}{ Original } & count & $0: 600$ & 5 & 0 & 0 & 0 & 0 & 5 \\
\hline & & $100: 500$ & 0 & 5 & 0 & 0 & 0 & 5 \\
\hline & & $300: 300$ & 0 & 0 & 5 & 0 & 0 & 5 \\
\hline & & 500:100 & 0 & 0 & 1 & 4 & 0 & 5 \\
\hline & & 600:0 & 0 & 0 & 0 & 0 & 5 & 5 \\
\hline \multirow[t]{5}{*}{ Cross-validated } & count & $0: 600$ & 5 & 0 & 0 & 0 & 0 & 5 \\
\hline & & $100: 500$ & 0 & 5 & 0 & 0 & 0 & 5 \\
\hline & & $300: 300$ & 0 & 0 & 5 & 0 & 0 & 5 \\
\hline & & 500:100 & 0 & 0 & 1 & 4 & 0 & 5 \\
\hline & & $600: 0$ & 0 & 0 & 0 & 0 & 5 & 5 \\
\hline
\end{tabular}

Table S13 Fluorescence response patterns generated from three sensor arrays against different concentrations of $\mathrm{Pb}^{2+}(10 \mu \mathrm{M}$ sensor in ultra-pure water $)$

$\mathrm{Pb}^{2+}(\mu \mathrm{M}) \quad$ Fold-change in fluorescence $(\mathrm{F})$ 


\begin{tabular}{|c|c|c|c|}
\hline & BER@Q[7] & H33342@2Q[7] & RhB@Q[7] \\
\hline 100 & 0.4480 & 0.9798 & 0.4888 \\
\hline 100 & 0.4183 & 0.9775 & 0.5052 \\
\hline 100 & 0.5312 & 0.9463 & 0.4864 \\
\hline 100 & 0.4287 & 0.9800 & 0.4861 \\
\hline 100 & 0.4205 & 0.9695 & 0.4755 \\
\hline 200 & 0.1553 & 0.9436 & 0.4516 \\
\hline 200 & 0.1471 & 0.9406 & 0.4475 \\
\hline 200 & 0.1278 & 0.9391 & 0.4468 \\
\hline 200 & 0.1389 & 0.9476 & 0.4482 \\
\hline 200 & 0.1360 & 0.9426 & 0.4370 \\
\hline 300 & 0.0810 & 0.9286 & 0.3926 \\
\hline 300 & 0.1003 & 0.9423 & 0.4025 \\
\hline 300 & 0.1122 & 0.9481 & 0.4097 \\
\hline 300 & 0.1055 & 0.9453 & 0.3820 \\
\hline 300 & 0.0906 & 0.9491 & 0.3844 \\
\hline 400 & 0.0602 & 0.9274 & 0.3698 \\
\hline 400 & 0.0490 & 0.9373 & 0.3326 \\
\hline 400 & 0.0498 & 0.9204 & 0.3657 \\
\hline 400 & 0.0617 & 0.9251 & 0.3380 \\
\hline 400 & 0.0646 & 0.9224 & 0.3459 \\
\hline 500 & 0.0557 & 0.9061 & 0.2671 \\
\hline 500 & 0.0275 & 0.9034 & 0.2323 \\
\hline 500 & 0.0624 & 0.9036 & 0.2316 \\
\hline 500 & 0.0475 & 0.9004 & 0.2435 \\
\hline 500 & 0.0423 & 0.9026 & 0.2105 \\
\hline 600 & 0.0342 & 0.9011 & 0.1862 \\
\hline 600 & 0.0208 & 0.8752 & 0.2006 \\
\hline
\end{tabular}




$\begin{array}{llll}600 & 0.0186 & 0.8667 & 0.2098 \\ 600 & 0.0171 & 0.8774 & 0.1798 \\ 600 & 0.0238 & 0.8747 & 0.2064\end{array}$

Unknown concentration(Actually $200 \mu \mathrm{M}$ )

$\begin{array}{llll}1 & 0.1478 & 0.9685 & 0.4526 \\ 2 & 0.1315 & 0.9531 & 0.4441 \\ 3 & 0.1278 & 0.9416 & 0.4127 \\ 4 & 0.1434 & 0.9301 & 0.4312 \\ 5 & 0.1434 & 0.9276 & 0.4199\end{array}$

Table S14 LDA classification characteristic values of three sensor array against different concentrations of $\mathrm{Pb}^{2+}(10 \mu \mathrm{M}$ sensor in ultra-pure water $)$

\begin{tabular}{ccccc}
\hline function & eigenvalues & Variation(\%) & Cumulative(\%) & Canonical correlation \\
\hline 1 & 438.568 & 96.4 & 96.4 & .999 \\
2 & 14.933 & 3.3 & 99.7 & .968 \\
3 & 1.450 & .3 & 100.0 & .769 \\
\hline
\end{tabular}

Table S15 Training matrix of fluorescence response patterns generated from three sensor arrays against different concentrations of $\mathrm{Pb}^{2+}(10 \mu \mathrm{M}$ sensor in ultra-pure water, red cells indicate false classifications $)$

\begin{tabular}{|c|c|c|c|c|c|c|c|c|c|}
\hline \multirow[t]{2}{*}{$\mathrm{Pb}^{2+}(\mu \mathrm{M})$} & \multicolumn{8}{|c|}{ Predictive classification } & \multirow[t]{2}{*}{ total } \\
\hline & & 0 & 100 & 200 & 300 & 400 & 500 & 600 & \\
\hline \multirow[t]{5}{*}{ Original } & 0 & 5 & 0 & 0 & 0 & 0 & 0 & 0 & 5 \\
\hline & 100 & 0 & 5 & 0 & 0 & 0 & 0 & 0 & 5 \\
\hline & 200 & 0 & 0 & 5 & 0 & 0 & 0 & 0 & 5 \\
\hline & 300 & 0 & 0 & 0 & 5 & 0 & 0 & 0 & 5 \\
\hline & 400 & 0 & 0 & 0 & 0 & 5 & 0 & 0 & 5 \\
\hline
\end{tabular}




\begin{tabular}{|c|c|c|c|c|c|c|c|c|c|}
\hline & 500 & 0 & 0 & 0 & 0 & 0 & 4 & 1 & 5 \\
\hline & 600 & 0 & 0 & 0 & 0 & 0 & 0 & 5 & 5 \\
\hline & Unknown & 0 & 0 & 4 & 1 & 0 & 0 & 0 & 5 \\
\hline \multirow{7}{*}{$\begin{array}{c}\text { Cross-val } \\
\text { idated }\end{array}$} & 0 & 5 & 0 & 0 & 0 & 0 & 0 & 0 & 5 \\
\hline & 100 & 0 & 5 & 0 & 0 & 0 & 0 & 0 & 5 \\
\hline & 200 & 0 & 0 & 5 & 0 & 0 & 0 & 0 & 5 \\
\hline & 300 & 0 & 0 & 0 & 5 & 0 & 0 & 0 & 5 \\
\hline & 400 & 0 & 0 & 0 & 0 & 5 & 0 & 0 & 5 \\
\hline & 500 & 0 & 0 & 0 & 0 & 0 & 4 & 1 & 5 \\
\hline & 600 & 0 & 0 & 0 & 0 & 0 & 0 & 5 & 5 \\
\hline
\end{tabular}

Table S16 Fluorescence response patterns generated from three sensor arrays against seven different metal ions (500 $\mu \mathrm{M}$ metal, $10 \mu \mathrm{M}$ sensor in Huaxi river water)

\begin{tabular}{|c|c|c|c|}
\hline \multicolumn{4}{|c|}{ Fold-change in fluorescence $(\mathrm{F})$} \\
\hline & BER@Q[7] & H33342@2Q[7] & RhB@Q[7] \\
\hline $\mathrm{Ba}^{2+}$ & 0.1096 & 0.3076 & 0.6569 \\
\hline $\mathrm{Ba}^{2+}$ & 0.1192 & 0.2736 & 0.5115 \\
\hline $\mathrm{Ba}^{2+}$ & 0.1401 & 0.3482 & 0.5868 \\
\hline $\mathrm{Ba}^{2+}$ & 0.1508 & 0.2508 & 0.3989 \\
\hline $\mathrm{Ba}^{2+}$ & 0.1753 & 0.2368 & 0.4675 \\
\hline $\mathrm{Hg}^{2+}$ & 0.9059 & 0.0142 & 0.9055 \\
\hline $\mathrm{Hg}^{2+}$ & 0.9477 & 0.0221 & 0.8902 \\
\hline $\mathrm{Hg}^{2+}$ & 1.0851 & 0.0146 & 0.8638 \\
\hline $\mathrm{Hg}^{2+}$ & 1.1621 & 0.0097 & 0.8332 \\
\hline $\mathrm{Hg}^{2+}$ & 0.9256 & 0.0233 & 0.8463 \\
\hline $\mathrm{Fe}^{2+}$ & 0.7332 & 1.7982 & 0.6405 \\
\hline $\mathrm{Fe}^{2+}$ & 0.6651 & 1.8566 & 0.6699 \\
\hline
\end{tabular}




\begin{tabular}{|c|c|c|c|}
\hline $\mathrm{Fe}^{2+}$ & 0.6580 & 1.9101 & 0.5708 \\
\hline $\mathrm{Fe}^{2+}$ & 0.6615 & 1.7207 & 0.6323 \\
\hline $\mathrm{Fe}^{2+}$ & 0.7123 & 1.7269 & 0.5961 \\
\hline $\mathrm{Fe}^{3+}$ & 0.1693 & 0.2699 & 0.0533 \\
\hline $\mathrm{Fe}^{3+}$ & 0.1914 & 0.3259 & -0.0030 \\
\hline $\mathrm{Fe}^{3+}$ & 0.1956 & 0.2649 & 0.0891 \\
\hline $\mathrm{fe}^{3+}$ & 0.1634 & 0.1858 & 0.0041 \\
\hline $\mathrm{fe}^{3+}$ & 0.1676 & 0.1775 & 0.0585 \\
\hline $\mathrm{Pb}^{2+}$ & 0.2619 & 2.8268 & 0.4802 \\
\hline $\mathrm{Pb}^{2+}$ & 0.2679 & 2.7621 & 0.5491 \\
\hline $\mathrm{Pb}^{2+}$ & 0.2840 & 2.6129 & 0.4902 \\
\hline $\mathrm{Pb}^{2+}$ & 0.1520 & 2.7779 & 0.5831 \\
\hline $\mathrm{Pb}^{2+}$ & 0.2374 & 2.6548 & 0.4034 \\
\hline $\mathrm{Al}^{3+}$ & 0.8055 & 1.5280 & 0.8004 \\
\hline $\mathrm{Al}^{3+}$ & 0.8318 & 1.3967 & 0.7318 \\
\hline $\mathrm{Al}^{3+}$ & 0.7995 & 1.4054 & 0.8921 \\
\hline $\mathrm{Al}^{3+}$ & 0.8396 & 1.5885 & 0.7751 \\
\hline $\mathrm{Al}^{3+}$ & 0.8897 & 1.6843 & 0.7818 \\
\hline $\mathrm{Cr}^{3+}$ & 0.9477 & 2.6970 & 0.1089 \\
\hline $\mathrm{Cr}^{3+}$ & 0.9280 & 2.6531 & 0.1883 \\
\hline $\mathrm{Cr}^{3+}$ & 0.9274 & 2.6249 & 0.2371 \\
\hline $\mathrm{Cr}^{3+}$ & 1.1239 & 2.6278 & 0.1693 \\
\hline $\mathrm{Cr}^{3+}$ & 0.9668 & 2.6109 & 0.1532 \\
\hline
\end{tabular}

Table S17 LDA classification characteristic values of three sensor array against seven different metal ions (500 $\mu \mathrm{M}$ metal, $10 \mu \mathrm{M}$ sensor in Huaxi river water)

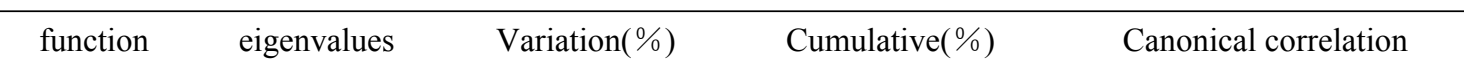




\begin{tabular}{lllll}
\hline 1 & 281.636 & 71.9 & 71.9 & .998 \\
2 & 95.720 & 24.4 & 96.3 & .995 \\
3 & 14.426 & 3.7 & 100.0 & .967 \\
\hline
\end{tabular}

Table S18 Training matrix of fluorescence response patterns generated from three sensor arrays against seven different metal ions $(500 \mu \mathrm{M}$ metal, $10 \mu \mathrm{M}$ sensor in Huaxi river water, red cells indicate false classifications)

\begin{tabular}{|c|c|c|c|c|c|c|c|c|c|c|}
\hline \multicolumn{2}{|l|}{ Metal } & \multicolumn{8}{|c|}{ Predictive classification } & \multirow[t]{2}{*}{ total } \\
\hline & & & $\mathrm{Ba}^{2+}$ & $\mathrm{Hg}^{2+}$ & $\mathrm{Fe}^{2+}$ & $\mathrm{Fe}^{3+}$ & $\mathrm{Pb}^{2+}$ & $\mathrm{Al}^{3+}$ & $\mathrm{Cr}^{3+}$ & \\
\hline \multirow[t]{7}{*}{ Original } & count & $\mathrm{Ba}^{2+}$ & 5 & 0 & 0 & 0 & 0 & 0 & 0 & 5 \\
\hline & & $\mathrm{Hg}^{2+}$ & 0 & 5 & 0 & 0 & 0 & 0 & 0 & 5 \\
\hline & & $\mathrm{Fe}^{2+}$ & 0 & 0 & 5 & 0 & 0 & 0 & 0 & 5 \\
\hline & & $\mathrm{Fe}^{3+}$ & 0 & 0 & 0 & 5 & 0 & 0 & 0 & 5 \\
\hline & & $\mathrm{Pb}^{2+}$ & 0 & 0 & 0 & 0 & 5 & 0 & 0 & 5 \\
\hline & & $\mathrm{Al}^{3+}$ & 0 & 0 & 0 & 0 & 0 & 5 & 0 & 5 \\
\hline & & $\mathrm{Cr}^{3+}$ & 0 & 0 & 0 & 0 & 0 & 0 & 5 & 5 \\
\hline \multirow[t]{7}{*}{ Cross-validated } & count & $\mathrm{Ba}^{2+}$ & 5 & 0 & 0 & 0 & 0 & 0 & 0 & 5 \\
\hline & & $\mathrm{Hg}^{2+}$ & 0 & 5 & 0 & 0 & 0 & 0 & 0 & 5 \\
\hline & & $\mathrm{Fe}^{2+}$ & 0 & 0 & 5 & 0 & 0 & 0 & 0 & 5 \\
\hline & & $\mathrm{Fe}^{3+}$ & 0 & 0 & 0 & 5 & 0 & 0 & 0 & 5 \\
\hline & & $\mathrm{Pb}^{2+}$ & 0 & 0 & 0 & 0 & 5 & 0 & 0 & 5 \\
\hline & & $\mathrm{Al}^{3+}$ & 0 & 0 & 0 & 0 & 0 & 5 & 0 & 5 \\
\hline & & $\mathrm{Cr}^{3+}$ & 0 & 0 & 0 & 0 & 0 & 0 & 5 & 5 \\
\hline
\end{tabular}

Table S19 Fluorescence response patterns generated from three sensor arrays against seven different metal ions (500 $\mu \mathrm{M}$ metal, $10 \mu \mathrm{M}$ sensor in Nongfu Spring mineral water)

Fold-change in fluorescence $(\mathrm{F})$ 


\begin{tabular}{|c|c|c|c|}
\hline & BER@Q[7] & H33342@2Q[7] & $\mathrm{RhB} @ \mathrm{Q}[7]$ \\
\hline $\mathrm{Ba}^{2+}$ & 0.0460 & 0.7619 & 0.1732 \\
\hline $\mathrm{Ba}^{2+}$ & 0.0253 & 0.8321 & 0.1809 \\
\hline $\mathrm{Ba}^{2+}$ & 0.0291 & 0.7318 & 0.1600 \\
\hline $\mathrm{Ba}^{2+}$ & 0.0352 & 0.8238 & 0.1968 \\
\hline $\mathrm{Ba}^{2+}$ & 0.0383 & 0.7286 & 0.1954 \\
\hline $\mathrm{Hg}^{2+}$ & 0.7745 & -0.0747 & 0.0118 \\
\hline $\mathrm{Hg}^{2+}$ & 0.7377 & -0.0748 & 0.0286 \\
\hline $\mathrm{Hg}^{2+}$ & 0.7293 & -0.0658 & 0.0695 \\
\hline $\mathrm{Hg}^{2+}$ & 0.7546 & -0.0739 & 0.0345 \\
\hline $\mathrm{Hg}^{2+}$ & 0.7400 & 0.0326 & 0.0382 \\
\hline $\mathrm{Fe}^{2+}$ & 0.3302 & 0.9689 & 0.3568 \\
\hline $\mathrm{Fe}^{2+}$ & 0.3240 & 0.8812 & 0.3827 \\
\hline $\mathrm{Fe}^{2+}$ & 0.3332 & 0.9265 & 0.4095 \\
\hline $\mathrm{Fe}^{2+}$ & 0.3279 & 1.0116 & 0.4181 \\
\hline $\mathrm{Fe}^{2+}$ & 0.3279 & 0.9353 & 0.4317 \\
\hline $\mathrm{Fe}^{3+}$ & 0.1279 & 0.2953 & 0.0141 \\
\hline $\mathrm{Fe}^{3+}$ & 0.1440 & 0.3018 & 0.0504 \\
\hline $\mathrm{Fe}^{3+}$ & 0.1295 & 0.2889 & 0.0718 \\
\hline $\mathrm{fe}^{3+}$ & 0.1440 & 0.2989 & 0.0995 \\
\hline $\mathrm{fe}^{3+}$ & 0.1410 & 0.2982 & 0.0205 \\
\hline $\mathrm{Pb}^{2+}$ & 0.0345 & 1.3267 & 0.1241 \\
\hline $\mathrm{Pb}^{2+}$ & 0.0299 & 1.5510 & 0.0868 \\
\hline $\mathrm{Pb}^{2+}$ & 0.0283 & 1.4203 & 0.1677 \\
\hline $\mathrm{Pb}^{2+}$ & 0.0460 & 1.3490 & 0.2145 \\
\hline $\mathrm{Pb}^{2+}$ & 0.0222 & 1.3117 & 0.2091 \\
\hline $\mathrm{Al}^{3+}$ & 0.8488 & 1.2804 & 0.6403 \\
\hline $\mathrm{Al}^{3+}$ & 0.8549 & 1.2617 & 0.6412 \\
\hline
\end{tabular}




$\begin{array}{llll}\mathrm{Al}^{3+} & 0.8748 & 1.2251 & 0.6113 \\ \mathrm{Al}^{3+} & 0.8472 & 1.2227 & 0.6562 \\ \mathrm{Al}^{3+} & 0.8710 & 1.2270 & 0.7435 \\ \mathrm{Cr}^{3+} & 0.8472 & 1.1054 & 0.2318 \\ \mathrm{Cr}^{3+} & 0.8587 & 1.0583 & 0.2195 \\ \mathrm{Cr}^{3+} & 0.8718 & 1.0639 & 0.2122 \\ \mathrm{Cr}^{3+} & 0.8396 & 1.1716 & 0.0795 \\ \mathrm{Cr}^{3+} & 0.8771 & 1.0639 & 0.1068\end{array}$

Table S20 LDA classification characteristic values of three sensor array against seven different metal ions (500 $\mu \mathrm{M}$ metal, $10 \mu \mathrm{M}$ sensor in Nongfu Spring mineral water)

\begin{tabular}{ccccc}
\hline function & eigenvalues & Variation(\%) & Cumulative(\%) & Canonical correlation \\
\hline 1 & 1674.699 & 89.9 & 89.9 & 1.000 \\
2 & 166.928 & 9.0 & 98.8 & .997 \\
3 & 21.492 & 1.2 & 100.0 & .978 \\
\hline
\end{tabular}

Table S21 Training matrix of fluorescence response patterns generated from three sensor arrays against seven different metal ions (500 $\mu \mathrm{M}$ metal, $10 \mu \mathrm{M}$ sensor in Nongfu Spring mineral water, red cells indicate false classifications)

\begin{tabular}{|c|c|c|c|c|c|c|c|c|c|c|}
\hline \multirow[t]{2}{*}{ Metal } & & \multicolumn{8}{|c|}{ Predictive classification } & \multirow[t]{2}{*}{ total } \\
\hline & & & $\mathrm{Ba}^{2+}$ & $\mathrm{Hg}^{2+}$ & $\mathrm{Fe}^{2+}$ & $\mathrm{Fe}^{3+}$ & $\mathrm{Pb}^{2+}$ & $\mathrm{Al}^{3+}$ & $\mathrm{Cr}^{3+}$ & \\
\hline \multirow[t]{6}{*}{ Original } & count & $\mathrm{Ba}^{2+}$ & 5 & 0 & 0 & 0 & 0 & 0 & 0 & 5 \\
\hline & & $\mathrm{Hg}^{2+}$ & 0 & 5 & 0 & 0 & 0 & 0 & 0 & 5 \\
\hline & & $\mathrm{Fe}^{2+}$ & 0 & 0 & 5 & 0 & 0 & 0 & 0 & 5 \\
\hline & & $\mathrm{Fe}^{3+}$ & 0 & 0 & 0 & 5 & 0 & 0 & 0 & 5 \\
\hline & & $\mathrm{Pb}^{2+}$ & 0 & 0 & 0 & 0 & 5 & 0 & 0 & 5 \\
\hline & & $\mathrm{Al}^{3+}$ & 0 & 0 & 0 & 0 & 0 & 5 & 0 & 5 \\
\hline
\end{tabular}




\begin{tabular}{ccccccccccc}
\hline & & $\mathrm{Cr}^{3+}$ & 0 & 0 & 0 & 0 & 0 & 0 & 5 & 5 \\
\hline Cross-validated & count & $\mathrm{Ba}^{2+}$ & 5 & 0 & 0 & 0 & 0 & 0 & 0 & 5 \\
& & $\mathrm{Hg}^{2+}$ & 0 & 5 & 0 & 0 & 0 & 0 & 0 & 5 \\
& $\mathrm{Fe}^{2+}$ & 0 & 0 & 5 & 0 & 0 & 0 & 0 & 5 \\
& $\mathrm{Fe}^{3+}$ & 0 & 0 & 0 & 5 & 0 & 0 & 0 & 5 \\
& $\mathrm{~Pb}^{2+}$ & 0 & 0 & 0 & 0 & 5 & 0 & 0 & 5 \\
& $\mathrm{Al}^{3+}$ & 0 & 0 & 0 & 0 & 0 & 5 & 0 & 5 \\
& $\mathrm{Cr}^{3+}$ & 0 & 0 & 0 & 0 & 0 & 0 & 5 & 5 \\
\hline
\end{tabular}

Table S22 Fluorescence response patterns generated from three sensor arrays against seven different metal ions (500 $\mu \mathrm{M}$ metal, $10 \mu \mathrm{M}$ sensor in Wahaha mineral water)

Fold-change in fluorescence $(\mathrm{F})$

\begin{tabular}{|c|c|c|c|}
\hline & BER@Q[7] & H33342@2Q[7] & RhB@Q[7] \\
\hline $\mathrm{Ba}^{2+}$ & 0.0300 & 0.9805 & 0.1768 \\
\hline $\mathrm{Ba}^{2+}$ & 0.0383 & 0.9849 & 0.1937 \\
\hline $\mathrm{Ba}^{2+}$ & 0.0248 & 0.9955 & 0.2038 \\
\hline $\mathrm{Ba}^{2+}$ & 0.0135 & 0.9964 & 0.2129 \\
\hline $\mathrm{Ba}^{2+}$ & 0.0345 & 0.9408 & 0.2149 \\
\hline $\mathrm{Hg}^{2+}$ & 0.7554 & -0.1357 & 0.0550 \\
\hline $\mathrm{Hg}^{2+}$ & 0.7884 & -0.1405 & 0.1179 \\
\hline $\mathrm{Hg}^{2+}$ & 0.7621 & -0.1350 & 0.1205 \\
\hline $\mathrm{Hg}^{2+}$ & 0.7546 & -0.1405 & 0.1035 \\
\hline $\mathrm{Hg}^{2+}$ & 0.7509 & 0.0241 & 0.1514 \\
\hline $\mathrm{Fe}^{2+}$ & 0.1659 & 0.4857 & 0.3364 \\
\hline $\mathrm{Fe}^{2+}$ & 0.2418 & 0.4616 & 0.3546 \\
\hline $\mathrm{Fe}^{2+}$ & 0.2831 & 0.4845 & 0.3696 \\
\hline $\mathrm{Fe}^{2+}$ & 0.3086 & 0.4813 & 0.2983 \\
\hline
\end{tabular}




\begin{tabular}{|c|c|c|c|}
\hline $\mathrm{Fe}^{2+}$ & 0.2012 & 0.4988 & 0.2989 \\
\hline $\mathrm{Fe}^{3+}$ & 0.1374 & 0.1396 & 0.0283 \\
\hline $\mathrm{Fe}^{3+}$ & 0.1719 & 0.1403 & 0.0335 \\
\hline $\mathrm{Fe}^{3+}$ & 0.1389 & 0.1320 & -0.0081 \\
\hline $\mathrm{fe}^{3+}$ & 0.1449 & 0.1633 & 0.1009 \\
\hline $\mathrm{fe}^{3+}$ & 0.1449 & 0.1224 & 0.0853 \\
\hline $\mathrm{Pb}^{2+}$ & 0.0518 & 0.9612 & 0.1810 \\
\hline $\mathrm{Pb}^{2+}$ & 0.0270 & 0.9504 & 0.1423 \\
\hline $\mathrm{Pb}^{2+}$ & 0.0255 & 0.9523 & 0.1563 \\
\hline $\mathrm{Pb}^{2+}$ & 0.0481 & 0.9589 & 0.1218 \\
\hline $\mathrm{Pb}^{2+}$ & -0.0023 & 0.9998 & 0.0736 \\
\hline $\mathrm{Al}^{3+}$ & 0.9093 & 0.9206 & 0.6483 \\
\hline $\mathrm{Al}^{3+}$ & 0.8830 & 0.8880 & 0.7082 \\
\hline $\mathrm{Al}^{3+}$ & 0.9161 & 0.9151 & 0.7362 \\
\hline $\mathrm{Al}^{3+}$ & 0.9138 & 0.9259 & 0.6984 \\
\hline $\mathrm{Al}^{3+}$ & 0.9476 & 0.9213 & 0.6948 \\
\hline $\mathrm{Cr}^{3+}$ & 0.8785 & 0.7968 & 0.2693 \\
\hline $\mathrm{Cr}^{3+}$ & 0.8733 & 0.7700 & 0.2667 \\
\hline $\mathrm{Cr}^{3+}$ & 0.8650 & 0.7406 & 0.2644 \\
\hline $\mathrm{Cr}^{3+}$ & 0.8522 & 0.7906 & 0.1892 \\
\hline $\mathrm{Cr}^{3+}$ & 0.8830 & 0.7794 & 0.2214 \\
\hline
\end{tabular}

Table S23 LDA classification characteristic values of three sensor array against seven different metal ions (500 $\mu \mathrm{M}$ metal, $10 \mu \mathrm{M}$ sensor in Wahaha mineral water)

\begin{tabular}{ccccc}
\hline function & eigenvalues & Variation(\%) & Cumulative(\%) & Canonical correlation \\
\hline 1 & 374.210 & 60.7 & 60.7 & .999 \\
2 & 209.394 & 34.0 & 94.7 & .998 \\
3 & 32.698 & 5.3 & 100.0 & .985 \\
\hline
\end{tabular}


Table S24 Training matrix of fluorescence response patterns generated from three sensor arrays against seven different metal ions $(500 \mu \mathrm{M}$ metal, $10 \mu \mathrm{M}$ sensor in Wahaha mineral water, red cells indicate false classifications)

\begin{tabular}{|c|c|c|c|c|c|c|c|c|c|c|}
\hline \multicolumn{2}{|l|}{ Metal } & \multicolumn{8}{|c|}{ Predictive classification } & \multirow[t]{2}{*}{ total } \\
\hline & & & $\mathrm{Ba}^{2+}$ & $\mathrm{Hg}^{2+}$ & $\mathrm{Fe}^{2+}$ & $\mathrm{Fe}^{3+}$ & $\mathrm{Pb}^{2+}$ & $\mathrm{Al}^{3+}$ & $\mathrm{Cr}^{3+}$ & \\
\hline \multirow[t]{7}{*}{ Original } & count & $\mathrm{Ba}^{2+}$ & 5 & 0 & 0 & 0 & 0 & 0 & 0 & 5 \\
\hline & & $\mathrm{Hg}^{2+}$ & 0 & 5 & 0 & 0 & 0 & 0 & 0 & 5 \\
\hline & & $\mathrm{Fe}^{2+}$ & 0 & 0 & 5 & 0 & 0 & 0 & 0 & 5 \\
\hline & & $\mathrm{Fe}^{3+}$ & 0 & 0 & 0 & 5 & 0 & 0 & 0 & 5 \\
\hline & & $\mathrm{Pb}^{2+}$ & 1 & 0 & 0 & 0 & 4 & 0 & 0 & 5 \\
\hline & & $\mathrm{Al}^{3+}$ & 0 & 0 & 0 & 0 & 0 & 5 & 0 & 5 \\
\hline & & $\mathrm{Cr}^{3+}$ & 0 & 0 & 0 & 0 & 0 & 0 & 5 & 5 \\
\hline \multirow[t]{7}{*}{ Cross-validated } & count & $\mathrm{Ba}^{2+}$ & 5 & 0 & 0 & 0 & 0 & 0 & 0 & 5 \\
\hline & & $\mathrm{Hg}^{2+}$ & 0 & 5 & 0 & 0 & 0 & 0 & 0 & 5 \\
\hline & & $\mathrm{Fe}^{2+}$ & 0 & 0 & 5 & 0 & 0 & 0 & 0 & 5 \\
\hline & & $\mathrm{Fe}^{3+}$ & 0 & 0 & 0 & 5 & 0 & 0 & 0 & 5 \\
\hline & & $\mathrm{Pb}^{2+}$ & 1 & 0 & 0 & 0 & 4 & 0 & 0 & 5 \\
\hline & & $\mathrm{Al}^{3+}$ & 0 & 0 & 0 & 0 & 0 & 5 & 0 & 5 \\
\hline & & $\mathrm{Cr}^{3+}$ & 0 & 0 & 0 & 0 & 0 & 0 & 5 & 5 \\
\hline
\end{tabular}

